\title{
Evaluation of Performance of Cloud of Things for Transferring Multimedia and Bulk set Data
}

\section{B Vivekanandam}

Senior Lecturer,

Faculty of Computer Science and Multimedia,

Lincoln University College, Malaysia.

Email: vivekanandam@lincoln.edu.my

\begin{abstract}
As the number of context-aware and unique data extracting and computing has grown leaps and bounds, paving way to their application in many platforms, Internet of Things (IoT) has gained a lot of importance in recent years. Using IoT, it is possible to connect any number of objects and in any context, giving rise to a diverse range of services. Hence power management, data storage, service discovery, service management, service creation and resource management need a more sophisticated mechanism and a better infrastructure. However, a single IoT that is power-constrained will not be able to tackle all the data that is generated. Hence cloud computing plays a crucial role by integrating with the IoT in such a way that the future internet and envisioned IoT is achievable. There are a number of challenges involved of which data trimming is common challenge. Due to unnecessary communication, the data this sent to the cloud may be interrupted. Hence to prevent this, data is initially preprocessed following which it is trimmed and then transferred to the cloud. This aspect of data processing can be carried out through Fog Computing or Smart Network that serves as a smart gateway. In this paper, we have introduced a novel concept Fog computing as the smart gateway. The results have been analysed and tested with respect to bulk-data synchronizstion Delay, bulk-data upload delay, jitter, synchronization delay and upload delay.
\end{abstract}

Keywords: Fog computing; smart gateway; cloud computing; IoT; smart gateway

\section{Introduction}

One of the most buzzing technology advancement in the recent decade is the development of internet of things which has led to a whole field of applications and equipment. This next 
Journal of Ubiquitous Computing and Communication Technologies (UCCT) (2021)

Vol.03/ No.01

Pages: $1-9$

https://www.irojournals.com/jucct/

DOI: https://doi.org/10.36548/jucct.2021.1.001

generation internet has paved way to making internet available, accessible and usable to people everywhere. Integration of cloud computing and IoT has also been introduced to meet the need for maintaining and updating the large amount of data that is gathered. In fact, in the near future, there will be thousands of devices connecting many people together using this technology [1]. A survey [2] shows that in the year 2012, the total amount of internet traffic generated by 20 households is equivalent to the whole internet usage in the year 2008. Apart from the introduction of internet, the invention of internet of things has accomplished itself as a household name. A large number of smart devices have also used the IoT to exchange information and data using IoT [3]. The vision of IoT is to encompass all the different aspects of life within the small smart devices that fall in our hands. Some of the typical smart devices are sensors, Arduino applications, mobile interfacing and so on. IoT proves to be the technological revolution that is will rule the market of reachability and connectivity in the future.

'Things' in IoT are used to refer to to any e object be it communicating or non communicating device. From a leaf in a tree to the functioning of a smart device anything could belong to the IoT [4]. Since internet is used as a medium for communication data transmission takes place through radio frequency identification RFID tags. Objects that use the digital environment and a part of the IoT are commonly referred to as smart objects. Hence, IoT is a combination of both software as well as hardware paradigm. Moreover, they also involve the social spectrum as well as the interacting aspects of the devices [5]-[6]. IoT also create multiple scenarios where it is necessary for devices to communicate between one another in order to pass information to the base node. The introduction of IoT has led to the revenue generation for new end users, vendors and manufacturers. Though IoT involves interaction between machines it is not necessarily confined to the same [7]. Using IoT entities can easily become a part of a larger system by means of a simple communicating device like RFID, bar-code and Bluetooth. Fig.1 represents the architecture of a typical 3-layer IoT having layers Application layer, Network layer and Perception layer [8]. Business layer and middleware layer are added to these layers, resulting in 5-layer architecture. 


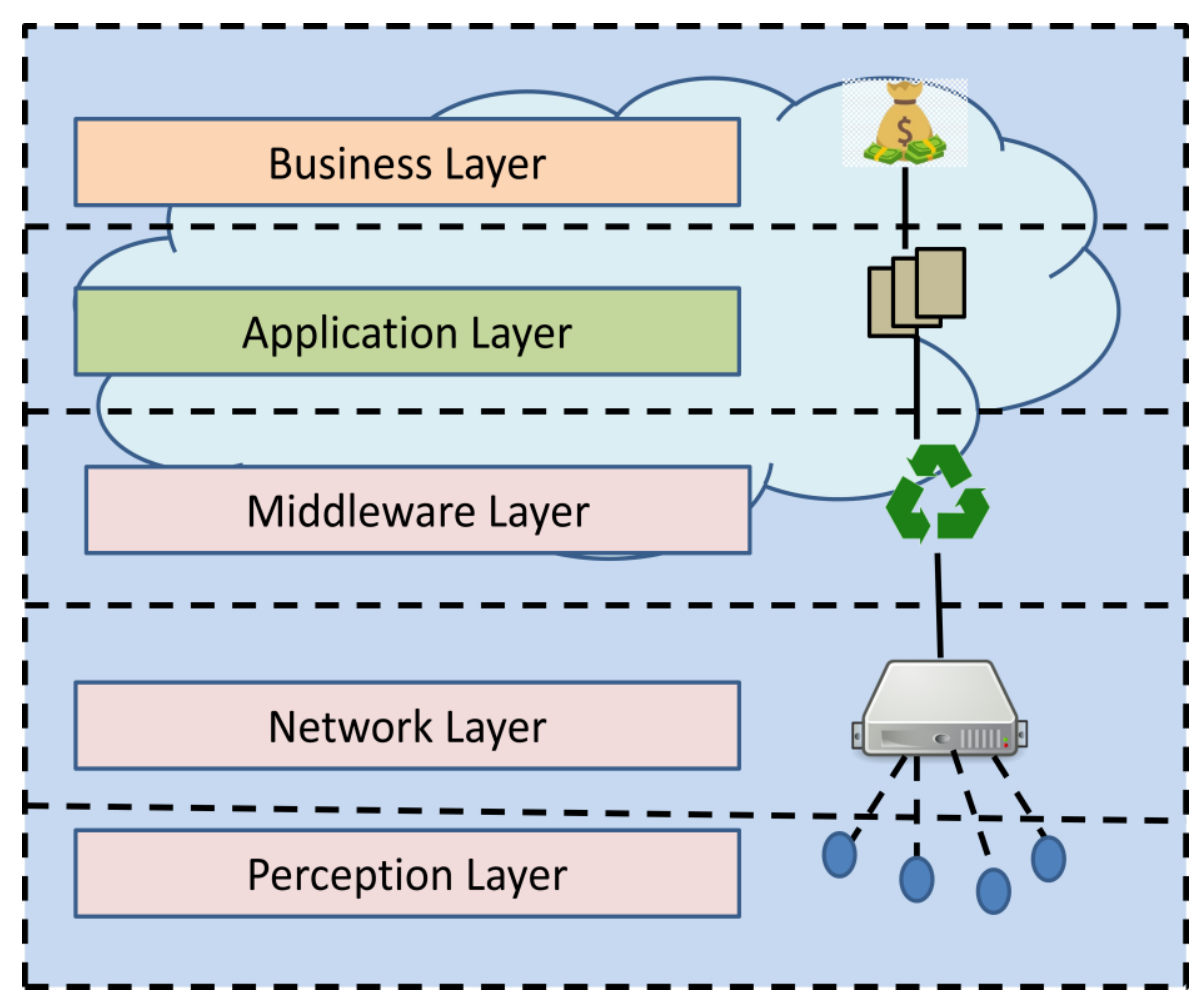

Fig.1. Architecture of Internet of Things Layers

The term computing which was confined to desktops has taken over the internet using cloud computing. The biggest advantage of this methodology is that the user doesn't have to worry about managing the resources or maintaining them [9]. The only thing that the user has to you think of is the cost of availing the service according to the type of cloud computing opted. A simple smart phone can serve as an interface to access vast amount of data through cloud computing. It is typically an extended form of grid computing parallel computing and distributed computing. Ubiquitous access to the internet and the data stored in it is possible by means of cloud computing and sharing this vast collection of of data is an additional feature provided [10]. In a distributed environment processing of the data is done in a hassle free manner using cloud computing. This research work presents the use of fog computing and smart gateway for smart communication known as cloud computing. 


\section{Proposed Work}

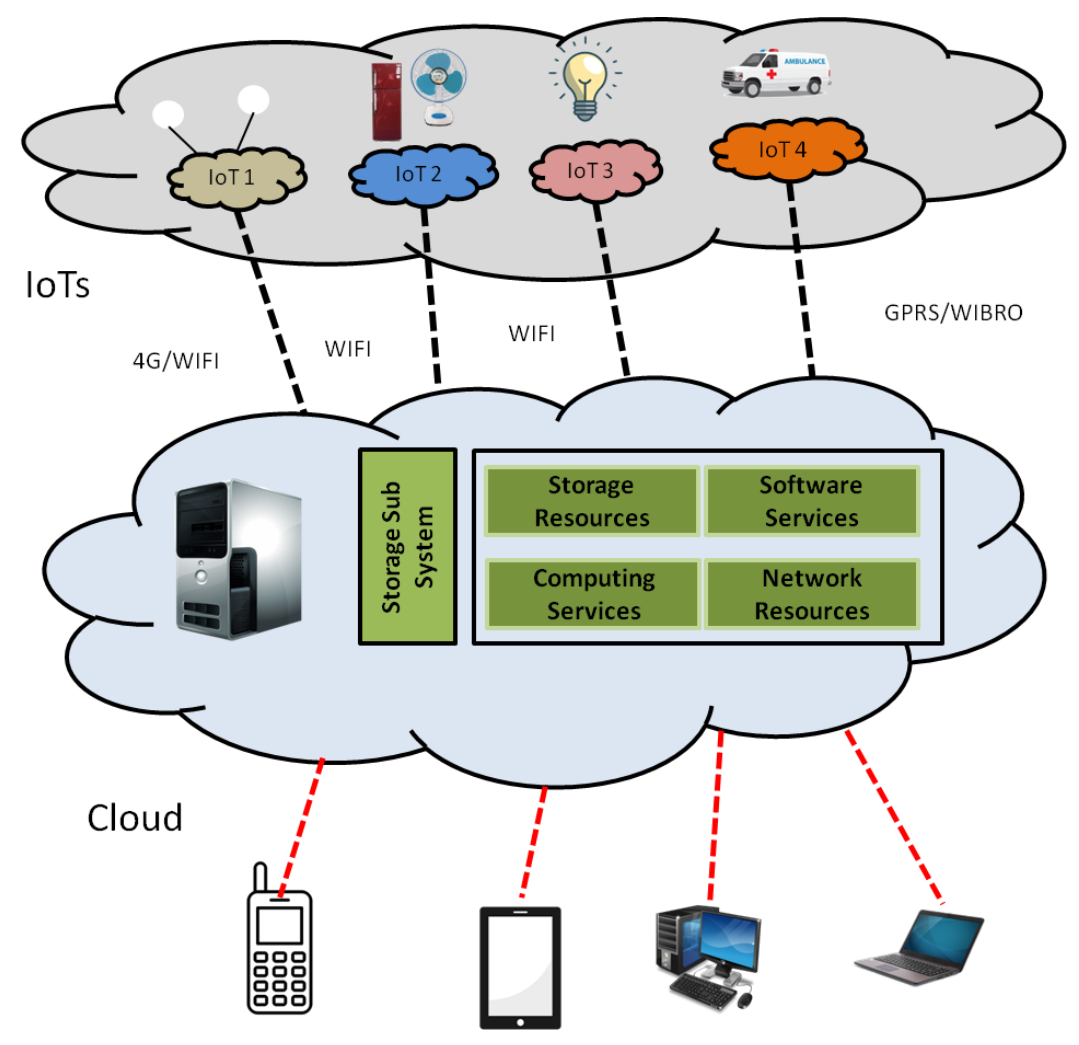

Fig.2 Cloud of Things

As we are progressing from the era of internet and internet of things, Cloud of Things [11] is a ubiquitous means of information exchange that is ruling the field of computing and data sorting. The total number of devices that are connected in 2020 is about 24 billion [12] and this number is bound to increase manifold in the next decade. This increase in the number of connected devices leads to the increase the data gathered and used [13][14]. This makes it almost impossible to save this data temporarily and locally and will also require a large amount of space. It is also crucial that the data that is gathered must be properly used, in the way that it deserves. The data gathered must be appropriately converted into knowledge that is used in further processing and mustn't be mere information. This requirement of processing information cannot be met by the IoT since it has constraints such as light-weight and cost consideration. This is where cloud computing will play a crucial role in integrating cloud computing and IoT to create a new environment of managing data and using it in an optimal manner by balancing the aspect of processing it, resulting in Cloud of Things (CoT). 
Journal of Ubiquitous Computing and Communication Technologies (UCCT) (2021)

Vol.03/ No.01

Pages: $1-9$

https://www.irojournals.com/jucct/

DOI: https://doi.org/10.36548/jucct.2021.1.001

This integration helps to manage the resources of the IoT in a better manner and will be effective. The introduction of CoT has given the users plenty of scope for using the devices as well as the services by making them accessible. This will also enable these services to be a monetary generation. Using CoT, analyzing the data generated by IoT as well as reacting on emergency and sensitive data is more effective. Fig.2 shows the overall architecture and communication protocol followed by a typical CoT. When it is possible to connect all the devices to the internet and use the devices based on their generation of data, a sync device or cloud is essential to keep track of the data. In such circumstances, the device must stop its basic activity of generating data. However, if a gateway device is fit between the device and the cloud, it will be useful in managing power in a more efficient form. Hence the gateway device that is placed in between should be able to perform some extra functions to send and receive data to and from the internet.

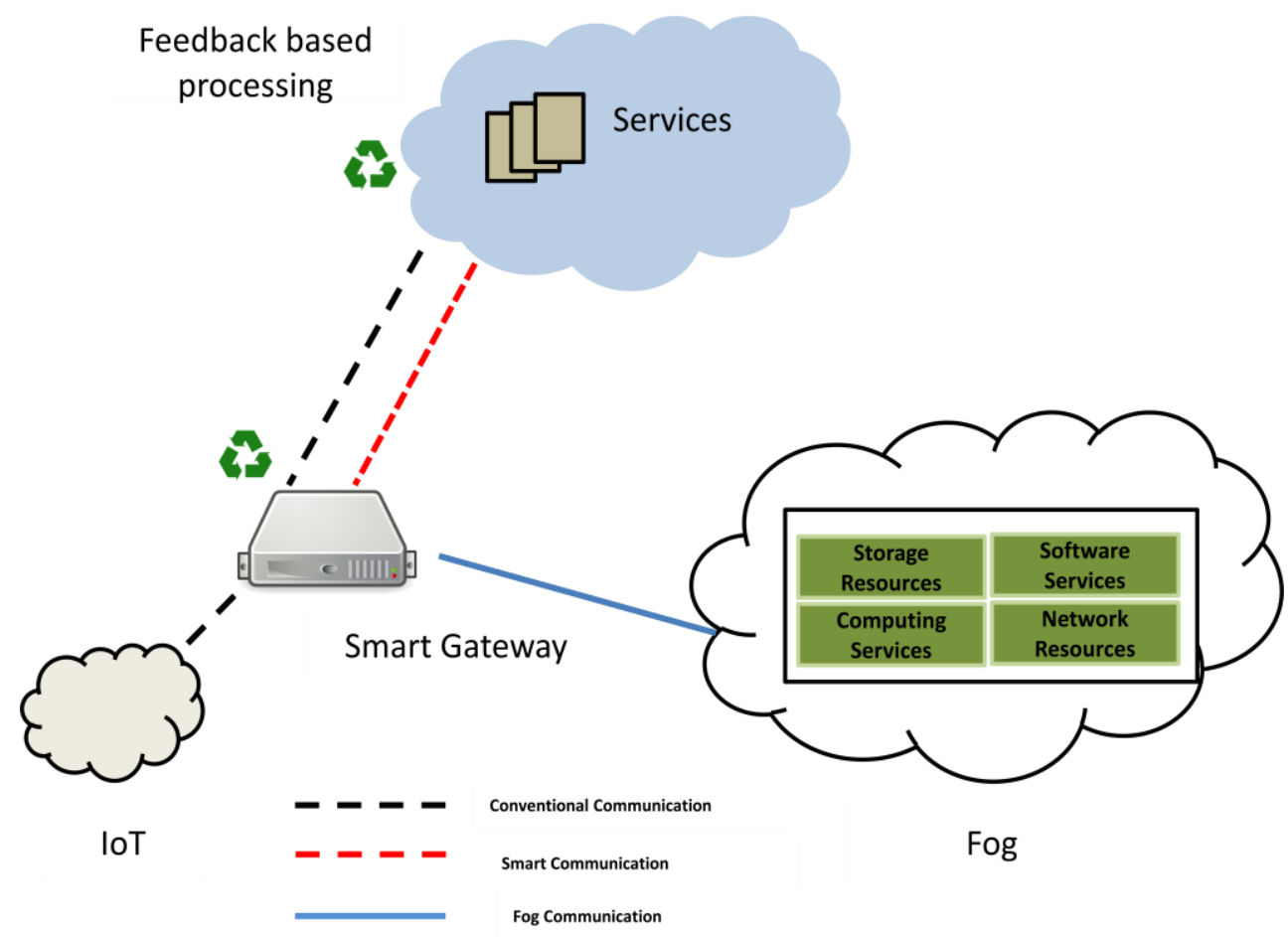

Fig.3. Smart Gateway using Smart Network

Depending on the application's feedback, it is important to decide on the type and timing of the data which is to be transmitted and this process is carried out through the 'Smart Gateway'. Keeping in mind these details, in this paper we have used a 6-layered architecture 
Journal of Ubiquitous Computing and Communication Technologies (UCCT) (2021)

Vol.03/ No.01

Pages: $1-9$

https://www.irojournals.com/jucct/

DOI: https://doi.org/10.36548/jucct.2021.1.001

of Smart Gateway. The layers used are virtual sensor networks, monitoring layer, preprocessing layer, temporary storage layer, security layer and transport layer.

- The transport layer is used to upload data onto the cloud, thereby reducing the processing space of the core.

- Data that is saved on the cloud might be sensitive and should not be affected by attacks, hence a proper privacy and security of data is essential which is provided by the security layer.

- The data management chores are performed using the preprocessing layer. At this layer, the data that is gathered undergoes the process of trimming, filtering to create the essential data.

- This processed data is then uploaded onto the cloud to be stored temporarily such that it can be accessed. When the stored data is no longer essential, it will be automatically deleted from the cloud storage.

- Monitoring layer is used to keep an eye on the different tasks of the underlying networks and nodes. Here, the node which is currently doing the task is monitored at this node. Similarly, the nodes or power constrained devices are also observed based on their energy consumption.

- The physical and virtualization layer represents the different sensors and devices that are connected to the IoT as 'Things'.

\section{Results and Discussion}

The performance evaluation of communication to and from the cloud and gateway are presented in this paper. Both bulk data as well as multimedia files are used as data sets. The bulk-data set is used comprises of various file types, sizes and formats while the multi-media data set is used with video and audio data. Uploading a $25 \mathrm{MB}$ video data onto the cloud can be done in about85 seconds. This is a typical average time value for the aspect of uploading video data. Similarly, relocation of data that is already present in the cloud will take about 4 seconds for synchronization delay. When transferring data, jitter plays a crucial role in affecting the information that is being transferred onto the cloud. Fig.44 represents the jitters that are recorded while Fig.6 indicates the standard deviation and variance of the jitter. When using bulk-set data, a total of 10MB was transferred and it took 30 seconds to upload while 
Journal of Ubiquitous Computing and Communication Technologies (UCCT) (2021)

Vol.03/ No.01

Pages: 1-9

https://www.irojournals.com/jucct/

DOI: https://doi.org/10.36548/jucct.2021.1.001

synchronization took 9 seconds which is almost mre than twice the time taken while sending $25 \mathrm{MB}$ of multimedia data.

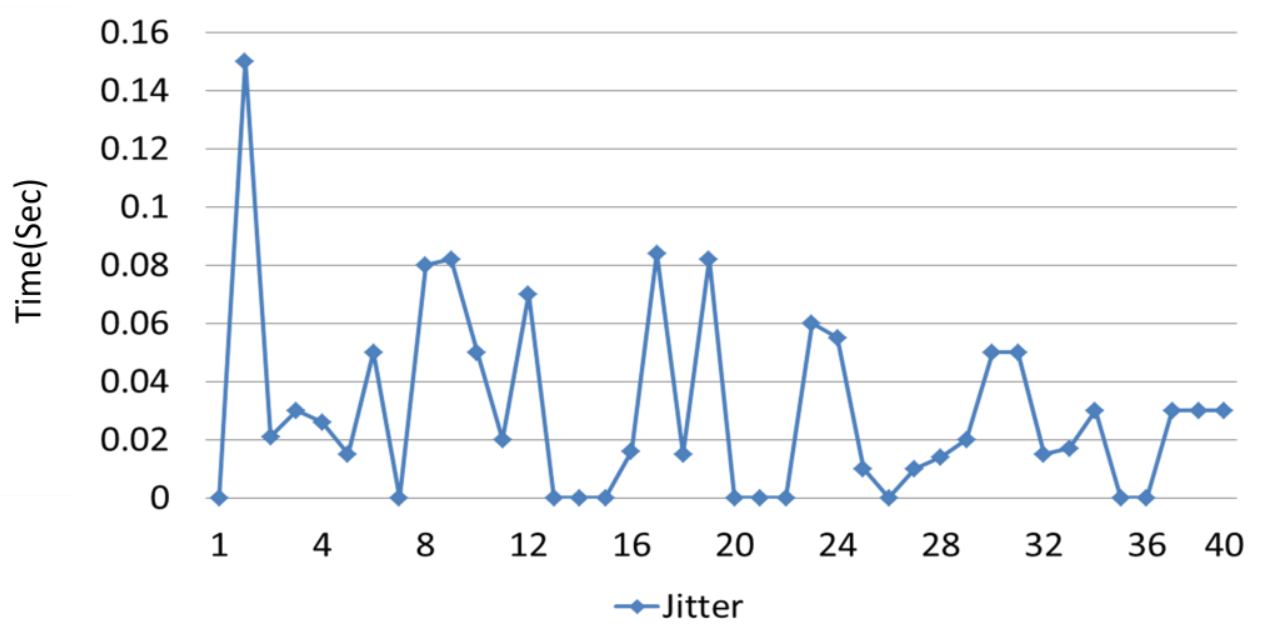

Packets

Fig.4. Jitter experienced using the Cloud

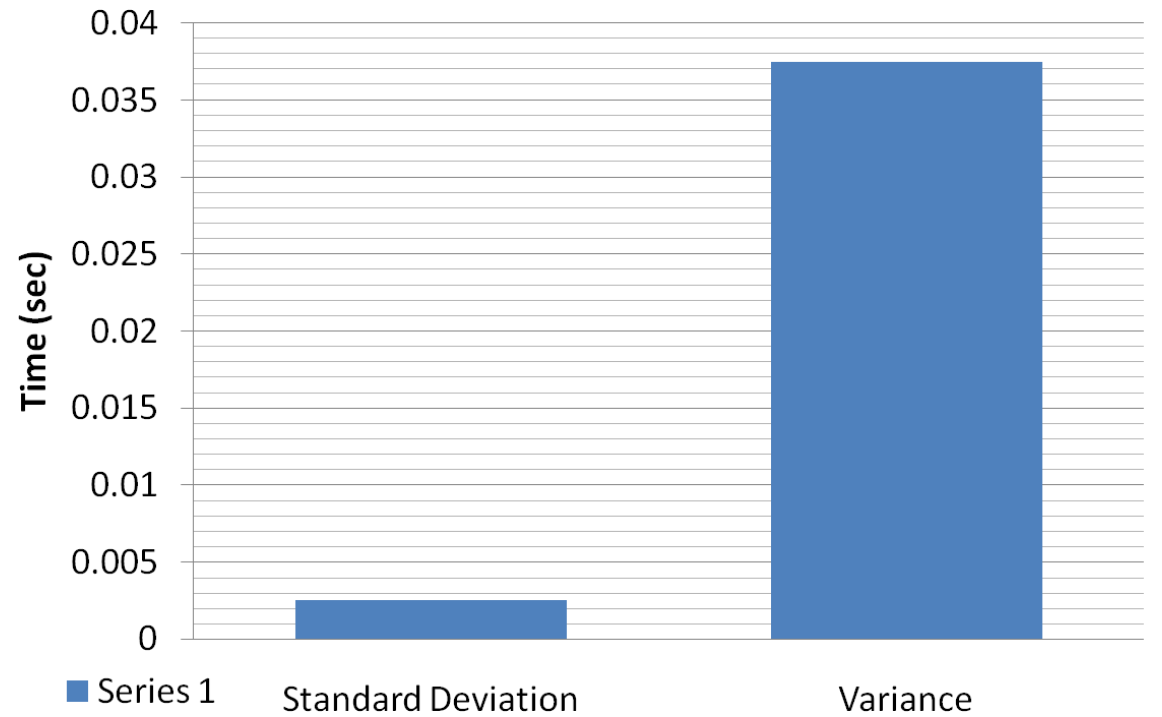

Fig.5 Standard Deviation and Variance for the Jitter observed

\section{Conclusion}

This paper shows the integration of cloud computing with IoT to provide a useful service with enhanced provisioning for efficient resource utilization. For prompt and better servicing, pre-processing and trimming of the data is essential before transmission to the 
Journal of Ubiquitous Computing and Communication Technologies (UCCT) (2021)

Vol.03/ No.01

Pages: $1-9$

https://www.irojournals.com/jucct/

DOI: https://doi.org/10.36548/jucct.2021.1.001

cloud. Hence we have introduced a smart gateway based means of transmission with the help of fog computing. This will enable the cloud to function better by providing a helping hand. This will also increase the overhead communication accomplished making it accessible by the cloud in order to build a better environment. This implementation is useful for delay sensitive applications where communication plays a crucial role. The integration of cloud computing with internet of Things and further involvement of Fog computing goes a long way in enhancing the way data collection, segregation and processing is performed. Depending on the different parameters used, a complete evaluation of the performance is also presented. Future work could involve the improvement of overall performance and heterogeneous storage depending on the type of application involved.

\section{References}

[1] Yannuzzi, M., Milito, R., Serral-Gracià, R., Montero, D., \& Nemirovsky, M. (2014, December). Key ingredients in an IoT recipe: Fog Computing, Cloud computing, and more Fog Computing. In 2014 IEEE 19th International Workshop on Computer Aided Modeling and Design of Communication Links and Networks (CAMAD) (pp. 325-329). IEEE.

[2] Zhang, H., Xiao, Y., Bu, S., Niyato, D., Yu, F. R., \& Han, Z. (2017). Computing resource allocation in three-tier IoT fog networks: A joint optimization approach combining Stackelberg game and matching. IEEE Internet of Things Journal, 4(5), 1204-1215.

[3] Suárez-Albela, M., Fernández-Caramés, T. M., Fraga-Lamas, P., \& Castedo, L. (2017). A practical evaluation of a high-security energy-efficient gateway for IoT fog computing applications. Sensors, 17(9), 1978.

[4] Sivaganesan, D. (2019). Design and development ai-enabled edge computing for intelligent-iot applications. Journal of trends in Computer Science and Smart technology (TCSST), 1(02), 84-94.

[5] Shirley, D. R. A., Janeera, D. A., Padmini, J. J., Banu, S. M. A., \& Abirami, T. (2018). Modelling and analysis of modified Baugh-Wooley multiplier using gate diffusion input and improved shannon adder. Int. J Pure Appl. Math, 118, 773-777.

[6] Al-Khafajiy, M., Baker, T., Waraich, A., Al-Jumeily, D., \& Hussain, A. (2018, December). IoT-fog optimal workload via fog offloading. In 2018 IEEE/ACM 
Journal of Ubiquitous Computing and Communication Technologies (UCCT) (2021)

Vol.03/ No.01

Pages: $1-9$

https://www.irojournals.com/jucct/

DOI: https://doi.org/10.36548/jucct.2021.1.001

International Conference on Utility and Cloud Computing Companion (UCC Companion) (pp. 359-364). IEEE.

[7] Alam, T. (2019). IoT-Fog: A communication framework using blockchain in the internet of things. Tanweer Alam." IoT-Fog: A Communication Framework using Blockchain in the Internet of Things.", International Journal of Recent Technology and Engineering (IJRTE), 7(6).

[8] Chang, Z., Liu, L., Guo, X., \& Sheng, Q. (2020). Dynamic resource allocation and computation offloading for IoT fog computing system. IEEE Transactions on Industrial Informatics.

[9] Sood, S. K., \& Mahajan, I. (2018). IoT-fog-based healthcare framework to identify and control hypertension attack. IEEE Internet of Things Journal, 6(2), 1920-1927.

[10] Kertész, A., Pflanzner, T., \& Gyimóthy, T. (2019). A mobile IoT device simulator for IoT-Fog-Cloud systems. Journal of Grid Computing, 17(3), 529-551.

[11] Tuli, S., Basumatary, N., \& Buyya, R. (2019, November). Edgelens: Deep learning based object detection in integrated iot, fog and cloud computing environments. In 2019 4th International Conference on Information Systems and Computer Networks (ISCON) (pp. 496-502). IEEE.

[12] Abbasi, M., Mohammadi-Pasand, E., \& Khosravi, M. R. (2021). Intelligent workload allocation in IoT-Fog-cloud architecture towards mobile edge computing. Computer Communications, 169, 71-80.

[13] Bestak, R., \& Smys, S. (2019). Big data analytics for smart cloud-fog based Applications. Journal of trends in Computer Science and Smart technology (TCSST), 1(02), 74-83.

[14] Shrestha, S., \& Shakya, S. (2020). A Comparative Performance Analysis of Fog-Based Smart Surveillance System. Journal of trends in Compu ter Science and Smart technology (TCSST) 2, (02), 78-88. 